Sitientibus Série Ciências Físicas 14: 1-13 (2018)

\title{
Duas Descrições Lagrangeanas para a Eletrodinâmica de London
}

\author{
Two Lagrangian Descriptions for the London's Electrodynamics
}

\author{
Edine Silva dos Santos e Franz A. Farias* \\ Departamento de Física - UEFS \\ Campus UEFS, Feira de Santana - BA - 44036-900
}

\begin{abstract}
A Eletrodinâmica de London é revisitada no contexto de duas descrições Lagrangianas. As equações de London descrevem o diamagnetismo extremo, característico dos supercondutores tipo I. Uma descrição Lagrangeana, em um primeiro caso, é estabelecida utilizando um multiplicador de Lagrange à Lagrangeana de Maxwell, enquanto na segunda descrição se utiliza a Lagrangeana de Proca. Mostramos que existe uma equivalência entre as duas Lagrangeanas e discutimos algumas das consequências dessa equivalência.
\end{abstract}

Palavras-chaves: Eletrodinâmica; Supercondutor; Equações de London; Fase Supercondutora; Comprimento de Penetração.

London Electrodynamics is revisited in the context of two Lagrangian descriptions. The London equations describe the extreme diamagnetism, characteristic of superconductors type I. We look for a Lagrangian description, in a first case using a Lagrange multiplier over Maxwell Lagrangian, and in a second one, using the Proca's Lagrangian. We show how the equivalence between the two Lagrangians is established and discuss some the consequences of these equivalence.

Keywords: Electrodynamics; Superconductor; London Equations; Superconducting Phase; Penetration Depth.

\section{INTRODUÇÃO}

O fenômeno da Supercondutividade completará, em 2021, 110 anos desde sua descoberta com a experiência realizada pelo físico holandês Kamerling Onnes sobre a resistência do mercúrio a baixíssimas temperaturas [1]

Heike Kamerlingh Onnes chefiava o mais avançado laboratório de criogenia da Europa no início do século XX. A descoberta da supercondutividade deveu-se, antes de tudo, à capacidade experimental para a liquefação do hélio líquido, e em geral, a liquefação dos chamados "gases permanentes". O hidrogênio já havia sido liquefeito no experimento realizado por Sir James Dewar, em 10 de maio de 1898. A liquefação do hélio era o próximo grande passo a ser alcançado, mas a dificuldade de obtenção de amostras puras de hélio bem como a própria técnica utilizada por De-

*Endereço Eletrônico: franz.farias@uefs.br war constituiram uma limitação nos resultados dos experimentos realizados [2] 4 .

A primeira liquefação efetiva do hélio foi conseguida em 10 de julho de 1908 por Kamerling Onnes e sua equipe, eles alcançaram a temperatura de 4, $25 \mathrm{~K}$ (veja a Figura 1). O sucesso impactante desse experimento [1] rendeu uma homenagem da equipe do laboratório ao Dr. Heike Kamerlingh Onnes, em seus 40 anos de seu professorado, em 11 de novembro de 1922 por seu feito em obter o hélio liquefeito!

Com o domínio da técnica de criogenia para obtenção do hélio líquido, este, por sua vez, passava a ser utilizado nos estudos experimentais das propriedades elétricas de metais. Digase de passagem, o interesse nesses estudos não iniciou em 1908 como se difunde grandemente, ao contrário, a investigação da relação entre resistência elétrica e temperatura foi proclamada em Leiden num anúncio oficial feito por Kamerlingh Onnes em 1902 [4, 5].

A evidência da queda brusca da resistivi- 
dade do mercúrio ocorreu em dezembro de 1911. A descoberta da supercondutividade dependeu do sucesso das técnicas criogênicas, e ambas foram possíveis no laboratório de baixas temperaturas na Universidade de Leiden (Holanda) [1, 3].

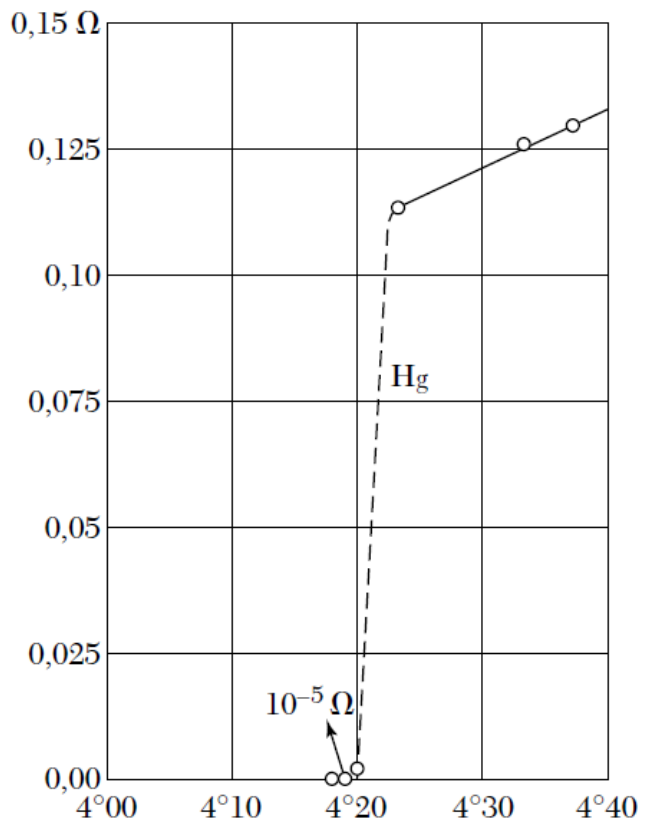

FIGURA 1: O gráfico mais conhecido em supercondutividade, trata-se do comportamento da resistividade do mercúrio e sua abrupta queda à tempetaura crítica de 4,25 K. Figura extraída da referência [6].

A primeira guerra mundial tornou muito difícil a comunicação entre os cientistas, e as novidades no campo do fenômeno da supercondutividade ficaram restritas à descoberta de novos metais e ligas supercondutoras. Somente em 1933 surgiria uma descoberta importante para a supercondutividade, o efeito Meissner-Ochsenfeld. Walther Meissner e Robert Ochsenfeld verificaram experimentalmente que os supercondutores eram diamagnetos extremos, ou seja, não havia campo magnético no interior do metal na fase supercondutora $\left(T<T_{c}\right)$. Esse comportamento independe da forma como se alcança a fase supercondutora 8-10], se baixando a temperatura primeiro e, em seguida, submetendo a amostra ao campo magnético, ou na ordem inversa.

A revelação do efeito Meissner-Ochsenfeld [11] encerrou a possibilidade de explicação do fenômeno da supercondutividade a partir da concepção apenas de um condutor perfeito com resistividade nula. As propriedade magnéticas de um supercondutor são, portanto, inteiramente distintas daquelas de um condutor perfeito. Podemos resumir o resultado experimental estabelecido por Meissner e Ochsenfeld pelo enunciado a seguir [12]:

"Um metal no estado supercondutor apresenta uma densidade de fluxo magnético sempre nula em seu interior."

Consequentemente, no interior de um metal supercondutor (entendido aqui como em sua fase supercondutora), temos sempre:

$$
\mathbf{B}=0 .
$$

Em 1935, surgiu a primeira tentativa bemsucedida de descrição da fase supercondutora pelo trabalho dos irmãos Heinz London e Fritz London. A medida experimental do comprimento de penetração do campo magnético era tema de trabalho de doutoramento de Heinz London. A teoria de London, como passou a ser conhecida [13, 14] tinha como principal objetivo explicar o resultado experimental para o efeito Meissner-Ochsenfeld [15-17.

Aspectos interessantes da teoria de London serão detalhados na primeira seção adiante, contudo a teoria de London é essencialmente clássica, e não conseguiu responder a questão sobre como surgiam os superelétrons. Efeitos quânticos serão levados em conta primeiramente pela teoria de Ginzburg-Landau, ou abreviadamente, Teoria GL, proposta por V.L. Ginzburg e L.D. Landau em 1950 [18]. A Teoria GL é a aplicação da teoria de transições de fase em $2^{a}$ ordem de Landau à supercondutividade [19], ela introduz um parâmetro de ordem com dependência espacial e a descrição da teoria é válida na vizinhança da temperatura crítica, $T_{c}-T \ll T_{c}$. Quando a densidade de superelétrons, $n_{s}$, atinge seu valor de equilíbrio em todo ponto no supercondutor, a Teoria GL reduz-se às equações de London [20]. 
A resposta sobre a natureza dos eletróns supercondutores, ou seja, sobre o mecanismo microscópico da supercondutividade, teria que aguardar até 1957, com o surgimento da primeira teoria quântica bem-sucedida a fornecer uma explicação para o fenômeno da supercondutividade com os pares de Cooper: a teoria BCS, de J. Bardeen, L. Cooper e R. Schrieffer, [21, 22, 24]. Essa teoria introduz novos aspectos a serem considerados na abordagem do fenômeno da supercondutividade, quando a frequência está acima da energia de gap (veja a nota em [23]). A supercondutividade se revelou, microscopicamente, como um fenômeno de excitações coletivas (a interação de pares de Cooper com os fónons, as vibrações da rede cristalina), a teoria BCS 25$]$.
Ainda no mesmo ano 1957, a descoberta dos supercondutores tipo II e a explicação de sua estrutura magnética pelo trabalho do físico Alexei A. Abrikosov [26], juntamente com Lev D. Landau, Vitaly Ginzburg e Zavaritsky [27], abrem um campo muito frutífero de pesquisas em supercondutividade. Os Supercondutores Tipo II apresentam um comportamento magnético diferente do Tipo I, pois agora o campo magnético penetra o material e o efeito Meissner-Ochsenfeld fica restrito à condição $\mathrm{H}<\mathrm{H}_{c 1}$. Ademais, a penetração do campo no material se manifesta de uma maneira não-usual, aparecem os padrões de fluxo de vórtices (veja a Figura 2 para os comportamentos de supercondutor tipo I e II).

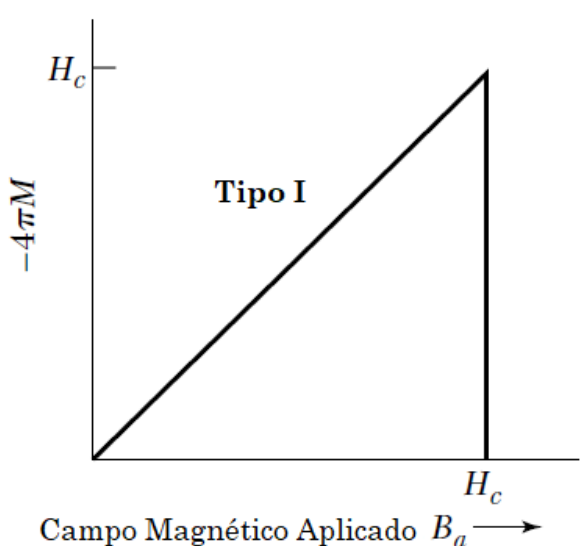

(a)

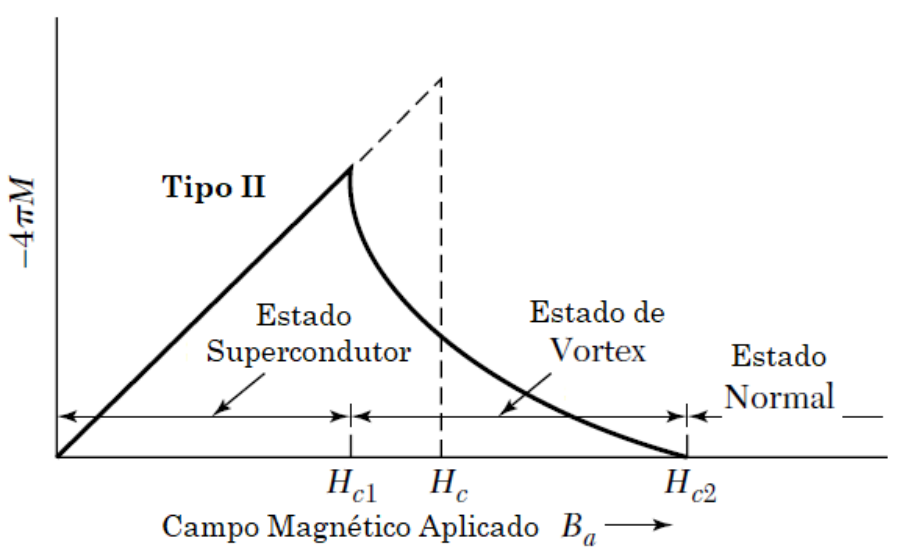

(b)

FIGURA 2: (a) Magnetização versus campo magnético aplicado para o volume de um supercondutor exibindo um efeito Meissner-Ochsenfeld perfeito (diamagnetismo perfeito). Um supercondutor com este comportamento é chamado um Supercondutor Tipo I. Acima do campo crítico $\mathrm{H}_{c}$ a amostra é um condutor normal e a magnetização é muito pequena para ser percebida nessa escala. Perceba que $(-4 \pi \mathrm{M})$ é plotado no eixo vertical: o valor negativo de $\mathrm{M}$ corresponde ao diamagnetismo. (b) a curva de magnetização supercondutora de um Supercondutor Tipo II. O fluxo começa a penetrar a amostra em um campo $\mathrm{H}_{c 1}$ abaixo do campo crítico termodinâmico $\mathrm{H}_{c}$. A amostra está no estado de vórtice entre $\mathrm{H}_{c 1}$ e $\mathrm{H}_{c 2}$, e tem propriedades elétricas supercondutoras até $\mathrm{H}_{c 2}$. Acima de $\mathrm{H}_{c 2}$ a amostra está no estado de condutor normal em todos os aspectos, exceto por possíveis efeitos de superfície. Figura e texto adaptados da referência 6 .

Os avanços seguintes em supercondutividade buscaram compostos e ligas com uma temperatura crítica de transição à fase supercondutora cada vez mais alta, é o campo da supercondutividade a altas temperaturas críticas. Exemplos desses compostos são ( $\mathrm{LaBa}$ ) $\mathrm{CuO}_{4}$, o primeiro composto cerâmico a exibir a supercondutividade em alta temperatura, $30 \mathrm{~K}$, descoberto em 1986, até o de mais alta temperatura já registrada, o com- 
posto $\mathrm{Cd}_{5} \mathrm{MgO}_{6}$, a $310 \mathrm{~K}$. Para um levantamento detalhado dos compostos e ligas em alta temperatura acesse a página da internet: http://superconductors.org. A área da supercondutividade já conta com 10 prêmios Nobel em Física (veja a referência [27]).

$\mathrm{Na}$ seção [II deste artigo, revemos a teoria de London no contexto da descrição lagrangiana, focando a fase supercondutora pura. Uma forma Lagrangeana condicionada é proposta, como uma Lagrangeana de London, e mostramos que ela é consistente com as equações da eletrodinâmica de London. Esta primeira lagrangeana incorpora um multiplicador de Lagrange associado a um vínculo à eletrodinâmica de Maxwell acoplado à corrente. Por sua vez, na seção III, abordamos a segunda densidade de Lagrangeana, a Lagrangeana de Proca, que descreve "fótons" massivos, mas agora voltada aos superelétrons. A relação entre as duas descrições é discutida no momento seguinte com um levantamento de implicações. Abordagens alternativas para as equações de London são discutidas na seção IV]. Ao final colocamos algumas observações na seção $\mathrm{V}$.

\section{A ELETRODINÂMICA DE LONDON}

A eletrodinâmica de London constitui uma extensão linear ad-hoc da eletrodinâmica de Maxwell [28] (em unidades gaussianas),

$$
\begin{aligned}
& \nabla \cdot \mathbf{D}(\mathbf{r}, t)=4 \pi \rho(\mathbf{r}, t) \\
& \nabla \cdot \mathbf{B}(\mathbf{r}, t)=0 \\
& \nabla \times \mathbf{E}(\mathbf{r}, t)+\frac{1}{c} \frac{\partial \mathbf{B}(\mathbf{r}, t)}{\partial t}=0, \\
& \nabla \times \mathbf{H}(\mathbf{r}, t)-\frac{1}{c} \frac{\partial \mathbf{D}(\mathbf{r}, t)}{\partial t}=\frac{4 \pi}{c} \mathbf{J}(\mathbf{r}, t),
\end{aligned}
$$

de maneira a descrever o fenômeno da supercondutividade a partir do diamagnetismo perfeito como expresso pela Eq.(1).

Vamos fazer uma breve digressão sobre a abordagem que adotaremos para o modelo do diamagnetismo perfeito. Como estamos interessados na distribuição espacial da indução magnética, B, a partir das equações de London, consideramos que o metal do mate- rial supercondutor é não-ferromagnético e nãomagnético, ou seja, a permeabilidade relativa é $\mu_{r}=1$, e que o material ao ser submetido à indução magnética externa, responde com uma indução magnética oposta devida às correntes de blindagem (induzidas), produzindo assim o cancelamento de $\mathbf{B}$ no interior do supercondutor (veja a subseção 2.2 .2 e o apêndice $A$ da referência [12] para maiores detalhes). Se estivéssemos interessados no estudo da energia de magnetização e do campo de demagnetização, seria mais apropriada a abordagem do diamagnetismo de volume [12].

Os irmãos Heinz e Fritz London propuseram em 1935 [13] duas equações adicionais, que em conjunto com as equações de Maxwell descrevem as propriedades eletromagnéticas de um supercondutor [12, 20, 29] (veja a nota em [30]), expressas como segue,

$$
\begin{aligned}
& \mathbf{E}=\frac{\partial \Lambda \mathbf{J}_{s}}{\partial t}, \\
& \mathbf{B}=-c \nabla \times \Lambda \mathbf{J}_{s},
\end{aligned}
$$

com o parâmetro fenomenológico $\Lambda$ dado por

$$
\Lambda=\frac{m}{n_{s} e^{2}},
$$

onde $m$ é a massa do elétron, $e$ é a carga do elétron, e $n_{s}$ é a densidade de superelétrons.

A Eq.(6) descreve a propriedade de resistência nula em um supercondutor, enquanto que a Eq. (7) descreve o diamagnetismo. A densidade de superelétrons $n_{s}$ compõe com a densidade de elétrons normais, $n_{N}$, a densidade total de elétrons livres $n=n_{N}+n_{s}$. Admitimos também, em conformidade com a abordagem acima descrita, que as correntes no metal afe$\operatorname{tam} \mathbf{B}$, mas não $\mathbf{H}$ e, por isto a Eq.(5) pode ser substituída por:

$$
\nabla \times \mathbf{B}-\frac{1}{c} \frac{\partial \mathbf{D}}{\partial t}=\frac{4 \pi}{c} \mathbf{J}_{s}
$$

Ademais, a variação temporal da corrente de deslocamento só é comparável a $\mathbf{J}_{s}$ quando os campos flutuam rapidamente com o tempo (veja a nota em [30]), logo (9) reduz-se a,

$$
\nabla \times \mathbf{B}=\frac{4 \pi}{c} \mathbf{J}_{s} .
$$


A Eq. (7) é uma consequência da Lei de Faraday em (4) com a equação de London em (6).

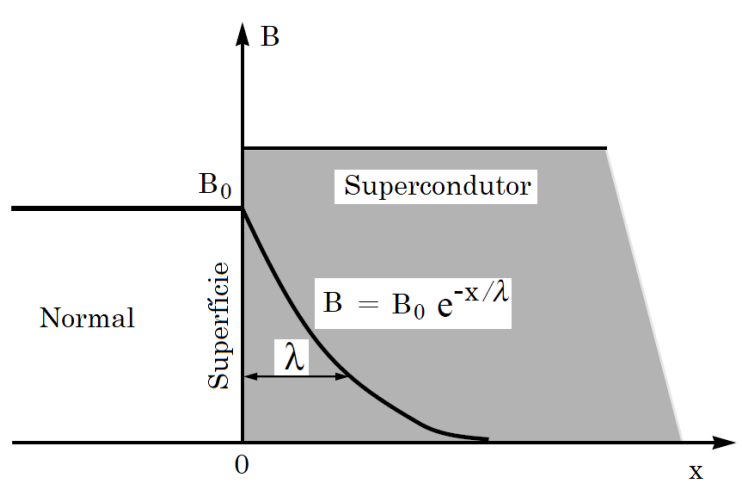

FIGURA 3: O comportamento de decaimento exponencial do campo magnético ao penetrar o material. Figura adaptada da referência [15].

Um condutor perfeito pensado como um gás de elétrons não-interagentes é consistente com a assertiva da Eq.(6), mas não com a Eq.(7), esta última é própria da corrente de superelétrons. Combinando (7) com (10), tendo em conta a identidade vetorial $\nabla \times \nabla \times \mathbf{B}=$ $\nabla(\nabla \cdot \mathbf{B})-\nabla^{2} \mathbf{B}$, obtemos para $\mathbf{B}$,

$$
\nabla^{2} \mathbf{B}=\frac{1}{\lambda_{L}^{2}} \mathbf{B}
$$

com o parâmetro $\lambda_{L}$ dado pela relação,

$$
\lambda_{L}^{2}=\frac{c^{2}}{4 \pi} \Lambda=\frac{m c^{2}}{4 \pi n_{s} e^{2}} .
$$

A Eq. (11) é o mais importante resultado decorrente das equações de London (6) e (7), e permite analisar o comportamento espacial do campo magnético, por exemplo, na situação de um campo magnético, $\mathrm{B}(\mathrm{x})$, paralelo à superfície da material. A solução, tendo em conta as condições de contorno $\mathrm{B}(\mathrm{x}=0)=B_{0} \mathrm{e}$ $\mathrm{B}(\mathrm{x} \rightarrow \infty)=0$, é neste caso,

$$
\mathrm{B}(\mathrm{x})=\mathrm{B}_{0} \mathrm{e}^{-\mathrm{x} / \lambda_{L}},
$$

onde $\mathrm{x}$ é medido a partir da superfície e $\lambda_{L}$ é o comprimento de penetração característico da material. Assim, podemos afirmar que o campo magnético é blindado no material após o comprimento de penetração $\lambda_{L}$.

A determinação de $\lambda_{L}$ depende da estimativa de $n_{s}$ que, por sua vez, pode ser conseguida identificando-a com a densidade efetiva de elétrons de condução nas medidas de impedância de superfície do comprimento de penetração no estado normal.

Uma predição teórica típica para $\lambda_{L}$ é da ordem de $200 \AA$, enquanto que os valores experimentais de $\lambda_{L}$ para amostras puras são da ordem de $500 \AA$ para $T \ll T_{c}$. Essa discrepância se explica por uma eletrodinâmica não-local em amostras supercondutoras puras. O resultado de London, decorrente de uma eletrodinâmica local, é consistente com os resultados experimentais para amostras supercondutoras cujos livres caminhos médios sejam curtos (veja a Tabela (I). A diferença observada entre teoria e experimento foi explicada pela primeira vez por Sir Alfred Pippard [32]. Em 1953, Pippard publicou sua eletrodinâmica não-local em analogia à proposta de generalização não-local da lei de Ohm estabelecida por Chambers.

A teoria de Pippard se baseou no conceito de comprimento de coerência, $\xi_{0}$, a partir da consideração que a função de onda para a fase supercondutora deveria possuir uma dimensão característica, uma vez que apenas elétrons com energia de Fermi da ordem de $K \mathrm{~T}_{c}$ desempenhariam papel relevante para a supercondutividade. O comprimento de coerência, $\xi_{0}$ poderia ser estimado, então, do princípio de incerteza de Heisenberg. Não discutiremos aqui os resultados de Pippard [33], pois foge ao escopo do presente trabalho.

Assim, embora na fase supercondutora tipo I, a ocorrência do efeito Meissner-Ochsenfeld defina o supercondutor como um diamagneto extremo, em experimentos o campo magnético penetra o material numa distância dada pelo parâmetro $\lambda_{L}$, o comprimento de penetração de London (veja a Figura 3). Esses fatos experimentais caracterizam o comportamento dos materiais designados por Supercondutores Tipo I [20, 34, e valem também para os Supercondutores Tipo II quando o campo magnético $\mathrm{H}<\mathrm{H}_{c 1}$ [12]. 
Theoretical Penetration Depth at $t=0, \lambda(0)$ compared with Experiment $\lambda_{0}$

\begin{tabular}{|c|c|c|c|c|c|c|}
\hline Metal & $\lambda_{L}(0) \times 10^{6}$ & $\xi_{0} \times 10^{6}$ & $\frac{\xi_{0}}{\lambda_{L}(0)}$ & $\frac{\lambda(0)}{\lambda_{L}(0)}$ & $\begin{array}{c}\lambda(0) \times 10^{6} \\
\text { (random } \\
\text { scattering) }\end{array}$ & $\begin{array}{c}\lambda(0) \times 10^{6} \\
\text { (observed) }\end{array}$ \\
\hline $\mathrm{Sn}$ & 3.4 & 23 & 6.2 & 1.5 & 5.1 & 5.1 \\
\hline $\mathrm{Al}$ & 1.6 & 160 & 100 & 3.3 & 5.3 & $4.9,5.15$ \\
\hline $\mathrm{Pb}$ & 3.7 & 8.3 & 2.2 & 1.25 & 4.4 & 3.9 \\
\hline $\mathrm{Cd}$ & 11.0 & 76 & 6.9 & 1.6 & 17.5 & 13 \\
\hline $\mathrm{Nb}$ & 3.9 & 3.8 & 0.98 & 1.15 & 4.5 & 4.4 \\
\hline
\end{tabular}

TABELA I: Valores teóricos e experimentais para o comprimento de penetração de London $\lambda_{L}$ de alguns metais e a comparação com o comprimento de coerência de Pippard, e outras razões entre estas grandezas. Figura extraída da referência [7].

Portanto, a eletrodinâmica de London decorre da hipótese fundamental que o diamagnetismo perfeito é a condição essencial justificando a persistência da corrente supercondutora no material, ao invés, como defendia Born e Heisenberg [25, da hipótese que uma corrente metaestável persistiria independentemente de um campo magnético aplicado.

\section{DESCRIÇÕES LAGRANGEANAS DA ELETRODINÂMICA DE LONDON}

Nosso objetivo é estabelecer as equações de London (6) e (7) a partir de uma densidade de Lagrangiana apropriadamente construída. Uma vez que, consideramos apenas a descrição da fase supercondutora e, portanto, não estamos analizando propriamente a transição entre fase normal e fase supercondutora (ou vice-versa) no material, o que necessariamente envolveria a dependência com a temperatura, podemos reduzir a abordagem a uma descrição Lagrangeana usual (puramente eletrodinâmica), dispensando assim a utilização da energia livre de Helmholtz, $\mathcal{F}(\mathbf{B}, \mathrm{T})$.

Utilizaremos a formulação covariante na abordagem da equivalência de descrições entre as densidades de Lagrangeanas de London e de Proca, pois essa escolha facilitará o estudo comparativo entre as duas descrições. A supercondutividade não é um fenômeno relativístico e poderia, em tese, ser descrito pela Mecânica Quântica não-Relativística, mas as características da supercondutividade se revelaram como uma manifestação de sistema de muitos corpos (um condensado, mas precisamente). O fenômeno da supercondutividade se mostrou desafiador aos físicos em suas buscas para estabelecer uma teoria a partir de primeiros princípios, e foram necessários 46 anos após a sua descoberta com Kamerling Onnes para que surgisse a teoria BCS.

\section{A. Descrição Lagrangeana da Eletrodinâmica de London}

Na formulação covariante, a dinâmica de um campo eletromagnético é descrita por uma densidade de Lagrangiana em termos do tensor de campo eletromagnético $\mathrm{F}_{\mu \nu}=\partial_{\mu} \mathrm{A}_{\nu}-\partial_{\nu} \mathrm{A}_{\mu}$, onde $\mathrm{A}_{\mu}$ é o quadripotencial eletromagnético,

$$
\mathrm{A}^{\mu} \equiv\left(\mathrm{A}_{0}, \mathbf{A}\right) .
$$

A densidade de corrente, $\mathbf{J}$, passa a ser composta por duas partes, uma densidade de corrente normal, $\mathbf{J}_{N}$, associada a $n_{N}$, e outra à densidade de superelétrons, $\mathbf{J}_{s}$, associada a $n_{s}$. 
A expressão para $\mathrm{F}_{\mu \nu}$ em forma matricial é,

$$
\begin{aligned}
\mathrm{F}^{\mu \nu} & \equiv \partial^{\mu} \mathrm{A}^{\nu}-\partial \nu \mathrm{A}^{\mu} \\
& =\left(\begin{array}{cccc}
0 & -\mathrm{E}_{\mathrm{x}} & -\mathrm{E}_{\mathrm{y}} & -\mathrm{E}_{\mathrm{z}} \\
\mathrm{E}_{\mathrm{x}} & 0 & -\mathrm{B}_{\mathrm{z}} & \mathrm{B}_{\mathrm{y}} \\
\mathrm{E}_{\mathrm{y}} & \mathrm{B}_{\mathrm{z}} & 0 & -\mathrm{B}_{\mathrm{x}} \\
\mathrm{E}_{\mathrm{z}} & -\mathrm{B}_{\mathrm{y}} & \mathrm{B}_{\mathrm{x}} & 0
\end{array}\right) .
\end{aligned}
$$

As equações de Maxwell, ou seja, da eletrodinâmica clássica, se escrevem em forma covariante como,

$$
\begin{aligned}
& \partial_{\mu} \mathrm{F}^{\mu \nu}=\frac{4 \pi}{c} \mathrm{~J}^{\nu}, \\
& \partial^{\alpha} \mathrm{F}^{\beta \gamma}+\partial^{\beta} \mathrm{F}^{\gamma \alpha}+\partial^{\gamma} \mathrm{F}^{\alpha \beta}=0,
\end{aligned}
$$

com a primeira equação obtida da densidade de Lagrangeana abaixo [28],

$$
\mathcal{L}_{M}\left[\mathrm{~A}^{\mu}\right]=-\frac{1}{16 \pi} \mathrm{F}_{\mu \nu} \mathrm{F}^{\mu \nu}-\frac{1}{c} \mathrm{~J}^{\mu} \mathrm{A}_{\mu} .
$$

A segunda equação, (17), não decorre de (18), constitui uma identidade. As equações (16) e (17) estão definidas no sistema gaussiano [28].

Propomos agora o vínculo a seguir,

$$
\Gamma\left(\mathrm{A}^{\mu}\right)=\left(\frac{1}{c} \mathrm{~A}_{\mu}+\Lambda \mathrm{J}_{s \mu}\right)\left(\frac{1}{c} \mathrm{~A}^{\mu}+\Lambda \mathrm{J}_{s}^{\mu}\right)=0 .
$$

Incorporando 19 com o multiplicador de Lagrange $\chi$ à Lagrangeana de Maxwell em 18 alcançamos a Lagrangeana de London, $\mathcal{L}_{L}$,

$$
\mathcal{L}_{L}\left[\mathrm{~A}^{\mu}, \chi\right]=\mathcal{L}_{M}+\chi \Gamma\left(\mathrm{A}^{\mu}\right),
$$

ou de outra forma,

$$
\begin{aligned}
& \mathcal{L}_{L}\left[\mathrm{~A}^{\mu}, \chi\right]=-\frac{1}{16 \pi} \mathrm{F}_{\mu \nu} \mathrm{F}^{\mu \nu}-\frac{1}{c} \mathrm{~J}_{s}^{\mu} \mathrm{A}_{\mu} \\
& +\chi\left(\frac{1}{c} \mathrm{~A}_{\mu}+\Lambda \mathrm{J}_{s \mu}\right)\left(\frac{1}{c} \mathrm{~A}^{\mu}+\Lambda \mathrm{J}_{s}^{\mu}\right),
\end{aligned}
$$

com $\mathrm{J}_{s}^{\mu} \equiv\left(0, \mathbf{J}_{s}\right)$. Ademais, lembramos que estamos admitindo unicamente a fase supercondutora pura, logo $\mathrm{J}_{N}^{\mu}=0$. Nos desenvolvimentos a seguir, já levaremos em conta esse fato.

A variação funcional em primeira ordem da ação associada, $\delta \mathcal{S}_{L}=0$, como estabelecido pelo Princípio de Hamilton,

$$
\delta S_{L}\left[\mathrm{~A}^{\mu}, \chi\right]=\delta \int \mathrm{d}^{4} \mathrm{x} \mathcal{L}_{L}=\int \mathrm{dt} \int \mathrm{d}^{3} \mathrm{x} \mathcal{L}_{L}
$$

impõe das equações de Euler-Lagrange,

$$
\frac{\partial \mathcal{L}_{L}}{\partial \mathrm{A}_{\mu}}-\partial_{\nu}\left(\frac{\partial \mathcal{L}_{L}}{\partial\left(\partial_{\nu} \mathrm{A}_{\mu}\right)}\right)=0,
$$

que sejam satisfeitas as equações,

$$
\begin{array}{ll}
\text { (I) } \quad & \frac{1}{4 \pi} \partial_{\alpha} \mathrm{F}^{\alpha \mu}+\frac{2}{c} \chi\left(\frac{1}{c} \mathrm{~A}^{\mu}+\Lambda \mathrm{J}_{s}^{\mu}\right) \\
-\frac{1}{c} \mathrm{~J}_{s}^{\mu}=0 & \\
\text { II }) \quad & \Gamma\left(\mathrm{A}^{\mu}\right)=0 \Rightarrow\left(\frac{1}{c} \mathrm{~A}^{\mu}+\Lambda \mathrm{J}_{s}^{\mu}\right)=0 .
\end{array}
$$

O vínculo que introduzimos em (19) é, naturalmente, o resultado por Euler-Lagrange em (22), que é a própria expressão da primeira equação de London em (6), agora escrita em termos do quadripotencial $\mathrm{A}^{\mu}$ dado por,

$$
\frac{1}{c} \mathrm{~A}^{\mu}=-\Lambda \mathrm{J}_{s}^{\mu} .
$$

Por sua vez, a Eq.21 pode ser simplificada, pois seu segundo termo, de acordo com a Eq. 222), é nulo, consequentemente,

$$
\partial_{\alpha} \mathrm{F}^{\alpha \mu}=\frac{4 \pi}{c} \mathrm{~J}_{s}^{\mu} .
$$

A equação acima resgata a lei de Ampère para a supercorrente, $\mathrm{J}_{s}^{\mu}$, em 10 , enquanto que da lei de Faraday e da primeira equação de London retomamos a segunda equação de London em (7). Naturalmente, estamos levando em conta que a contribuição devida à corrente de deslocamento é desprezível (veja maiores detalhes nos comentários da nota [31]).

Vamos explicitar os termos na Lagrangeana em 20) para investigar o vínculo $\chi$,

$$
\begin{aligned}
\mathcal{L}_{L}\left[\mathrm{~A}^{\mu}, \chi\right] & =-\frac{1}{16 \pi} \mathrm{F}_{\mu \nu} \mathrm{F}^{\mu \nu}-\frac{1}{c} \mathrm{~J}_{s}^{\mu} \mathrm{A}_{\mu} \\
& +\frac{1}{c^{2}} \chi \mathrm{A}_{\mu} \mathrm{A}^{\mu}+\frac{2}{c}(\chi \Lambda) \mathrm{J}_{s}^{\mu} \mathrm{A}_{\mu} \\
& +\chi \Lambda^{2} \mathrm{~J}_{s \mu} \mathrm{J}_{s}^{\mu} .
\end{aligned}
$$

O último termo na Lagrangeana acima é, para 
todos os efeitos, um termo constante. Com isso, podemos construir uma densidade de Lagrangeana modificada, descartando esse termo constante, uma vez que o mesmo não afeta as equações dinâmicas, assim,

$$
\begin{aligned}
\mathcal{L}_{L}^{\prime}\left[\mathrm{A}^{\mu}, \chi\right] & =-\frac{1}{16 \pi} \mathrm{F}_{\mu \nu} \mathrm{F}^{\mu \nu}+\frac{1}{c^{2}} \chi \mathrm{A}_{\mu} \mathrm{A}^{\mu} \\
& -\frac{1}{c}(1-2 \chi \Lambda) \mathrm{J}_{s}^{\mu} \mathrm{A}_{\mu}
\end{aligned}
$$

Temos, então, a escolha de tornar nulo o último termo em 26), definindo $\chi$ como,

$$
\chi=(2 \Lambda)^{-1}=\frac{c^{2}}{8 \pi \lambda_{L}^{2}} .
$$

A forma final da Lagrangeana $\mathcal{L}_{L}^{\prime}$ é então,

$$
\mathcal{L}_{L}^{\prime}\left[\mathrm{A}^{\mu}\right]=-\frac{1}{16 \pi} \mathrm{F}_{\mu \nu} \mathrm{F}^{\mu \nu}+\frac{1}{8 \pi \lambda_{L}^{2}} \mathrm{~A}_{\mu} \mathrm{A}^{\mu} .
$$

Na próxima subseção, mostraremos que a teoria de London pode, também, ser alcançada a partir de uma densidade de Lagrangeana de Proca e, em seguida, como a Lagrangeana em 28) se relaciona com a de Proca.

\section{B. A Lagrangiana de Proca para a Teoria de London}

Em um artigo de 1935 [35, 36, Hideki Yukawa lançou a hipotése de um mediador massivo para a interação nuclear forte em estreita analogia com o fóton da interação eletromagnética. A razão de uma massa para o mediador da interação nuclear forte é seu curto alcance (até $15 \mathrm{fm}$ ) comparado ao da interação eletromagnética (infinito). Esse mediador, inicialmente denominado como mésotron, passou a ser designado por méson e teve sua existência comprovada em 1947 através dos experimentos com os raios cósmicos conduzidos pela equipe de Bristol, da qual fazia parte o físico brasileiro César Lattes [37]. Em 1949, Yukawa foi agraciado com o Prêmio Nobel de Física, e Cecil Frank Powell, o chefe da equipe de Bristol, com o Prêmio Nobel de Física de 1950.

Uma das descrições para o campo do méson foi estabelecida em (1936) por Alexandru Proca
[38 40]. Ele propôs uma equação para descrever o campo bosônico vetorial massivo, estendendo as equações de Maxwell ao contexto da Teoria Quântica de Campos através de uma densidade de Lagrangeana relativística com um termo de massa.

O nome de Proca foi citado por Wolfgang Pauli em sua palestra do Prêmio Nobel, em 13 de dezembro de 1946, como o autor da equação que descreve campos bosônicos massivos, e a Lagrangiana associada foi denominada, a partir de então, por Lagrangiana de Proca.

Nosso objetivo é mostrar que a supercondutividade, em seu aspecto eletrodinâmico no contexto das equações de London, pode ser descrita também em termos de uma densidade de Lagrangeana de Proca, considerando que no supercondutor os campos são dotados de uma massa de "fóton" efetiva.

A densidade de Lagrangeana de Proca original tem a seguinte forma [28],

$\mathcal{L}_{P}\left[\mathrm{~A}^{\alpha}\right]=-\frac{1}{16 \pi} \mathrm{F}_{\alpha \beta} \mathrm{F}^{\alpha \beta}-\frac{1}{c} \mathrm{~A}_{\alpha} \mathrm{J}_{P}^{\alpha}+\frac{\eta^{2}}{8 \pi} \mathrm{A}_{\alpha} \mathrm{A}^{\alpha}$,

onde o parâmetro $\eta$ tem dimensões de inverso de comprimento e é o recíproco do comprimento de onda de Compton do fóton,

$$
\eta=\frac{m_{\gamma} c}{\hbar} .
$$

As equações de movimento de Proca são:

$$
\partial_{\beta} \mathrm{F}^{\beta \alpha}+\eta^{2} \mathrm{~A}^{\alpha}=\frac{4 \pi}{c} \mathrm{~J}_{P}^{\alpha} .
$$

Explicitanto o tensor de campo eletromagnético, $\mathrm{F}^{\beta \alpha}$, em função do quadripotencial,

$$
\partial_{\beta} \partial^{\beta} \mathrm{A}^{\alpha}-\partial^{\alpha}\left(\partial_{\beta} \mathrm{A}^{\beta}\right)+\eta^{2} \mathrm{~A}^{\alpha}=\frac{4 \pi}{c} \mathrm{~J}_{P}^{\alpha},
$$

e considerando o calibre de Lorentz, $\partial_{\mu} \mathrm{A}^{\mu}=0$, resulta

$$
\partial_{\beta} \partial^{\beta} \mathrm{A}^{\alpha}+\eta^{2} \mathrm{~A}^{\alpha}=\frac{4 \pi}{c} \mathrm{~J}_{P}^{\alpha},
$$

ou ainda, em termos do potencial vetor,

$$
\triangle \mathbf{A}-\frac{1}{c^{2}} \frac{\partial^{2} \mathbf{A}}{\partial t^{2}}-\eta^{2} \mathrm{~A}^{\alpha}=-\frac{4 \pi}{c} \mathrm{~J}_{P}^{\alpha} .
$$


Portanto, a equação dinâmica de um campo massivo, associado a $\mathbf{A}$, na presença da fonte $\mathrm{J}_{P}^{\alpha}$. Vamos agora estabelecer a densidade de Lagrangeana de Proca para um termo de massa que seja correspondente a $\eta \equiv \eta_{L}$. Para proceder à comparação vamos reescrever a densidade de Lagrangeana de Proca em 29),

$$
\begin{aligned}
\mathcal{L}_{P}\left[\mathrm{~A}^{\mu}\right] & =-\frac{1}{16 \pi} \mathrm{F}_{\mu \nu} \mathrm{F}^{\mu \nu}+\frac{1}{c} \mathrm{~A}_{\mu} \mathrm{J}_{P}^{\mu} \\
& +\frac{\eta_{L}}{8 \pi} \mathrm{A}_{\mu} \mathrm{A}^{\mu}
\end{aligned}
$$

A comparação entre as Lagrangeanas (28) e (33) revela as identidades:

$$
\eta_{L}=\lambda_{L}^{-1}, \quad \mathrm{~J}_{P}^{\mu}=0
$$

O parâmetro $\eta_{L}$ no termo associado à massa em Proca, tem dimensão de inverso de comprimento, e está agora associado ao inverso do comprimento de penetração de London, $\lambda_{L}$.

Numa reinterpretação do resultado em (28), podemos dizer que a densidade de Lagrangeana de London, construída a partir da Lagrangeana de Maxwell com vínculo, constitui uma Lagrangeana de Proca sem fontes.

\section{DEDUÇÕES ALTERNATIVAS PARA AS EQUAÇÕES DE LONDON}

Até a subseção anterior, abordamos as equações de London considerando a energia do sistema campo e supercondutor como armazenada no campo. Assim, construímos densidades de Lagrangeana de campo (London e Proca) em interação com a supercorrente de onde seguiu-se as equações de London.

Nesta seção, alternamos a abordagem da energia para considerá-la como armazenada no sistema de partículas, ou seja, associada à corrente dos superelétrons. A Lagrangeana do sistema de superelétrons na presença de um campo eletromagnético externo é,

$$
\tilde{\mathrm{L}}=\mathrm{K}_{s}+\frac{e}{c} n_{s} \mathbf{v}_{s} \cdot \mathbf{A}-e \varphi
$$

onde $\mathrm{K}_{s}$ é a energia cinética associada aos superelétrons. Ademais, lembramos as relações entre os campos e os potenciais escalar e vetor,

$$
\mathbf{E}=-\nabla \varphi-\frac{1}{c} \frac{\partial \mathbf{A}}{\partial t}, \quad \mathbf{B}=\nabla \times \mathbf{A}
$$

O ponto de partida é o acoplamento mínimo, e para tanto precisamos do momento canônico, $\mathbf{p}_{s}$ cuja expressão da Lagrangiana em (35) é,

$$
\mathrm{p}_{s j}=\frac{\partial \tilde{\mathrm{L}}}{\partial \mathrm{v}_{s j}}=n_{s} m \mathrm{v}_{s j}+\frac{e}{c} \mathrm{~A}_{j}
$$

com u sendo a velocidade dos superelétrons, ou ainda reescrevendo (37),

$$
\mathbf{p}_{s}=n_{s} m \mathbf{v}_{s}+\frac{e}{c} \mathbf{A} .
$$

Note que $\mathbf{p}_{s}$ é dependente do calibre, mas o momento mecânico não.

\section{A. As Equações de London a partir do Acoplamento Mínimo}

O vínculo em (19), que compôs a Lagrangeana de London, tem uma forma covariante, mas dependente da escolha de calibre. Para entender a implicação disso, vamos mostrar como as equações de London seguem do momento canônico [20, 29].

A densidade de corrente de superelétrons é,

$$
\mathbf{J}_{s}=n_{s} e \mathbf{v}_{s},
$$

e com isso a Eq. $(38)$ pode ser reescrita,

$$
\mathbf{p}_{s}=\frac{e}{c} \mathbf{A}+e \Lambda \mathbf{J}_{s},
$$

com $\Lambda$ dado por (8). Se impusermos que o momento canônico seja irrotacional,

$$
\nabla \times \mathbf{p}_{s}=0,
$$

resgatamos a segunda equação de London em 77). Por outro lado, efetuando a derivada total no tempo da relação 40 obtemos,

$$
\begin{aligned}
\dot{\mathbf{p}}_{s} & \equiv \frac{d \mathbf{p}_{s}}{d t}=n_{s} \frac{e}{c}\left[\nabla \cdot \mathbf{A}+\frac{\partial \mathbf{A}}{\partial t}\right] \\
& +n_{s} e\left[\nabla \cdot\left(\Lambda \mathbf{J}_{s}\right)+\frac{\partial\left(\Lambda \mathbf{J}_{s}\right)}{\partial t}\right] .
\end{aligned}
$$


$\mathrm{Na}$ expressão acima, admitindo que $\dot{\mathbf{p}}_{s}=0$, considerando o calibre de Coulomb, $\nabla \cdot \mathbf{A}=0$, e que $\mathbf{J}_{s}$ seja estacionária, $\nabla \cdot \mathbf{J}_{s}=0$, resulta a primeira equação de London, (6).

Portanto, a constância no tempo do vetor momento canônico implica na expressão do vínculo (19), mas para conseguirmos a equação que dá conta do efeito Meissner-Ochsenfeld precisamos impor (41). Ademais, o calibre de Coulomb deve ser escolhido e a Eq.41) traz o superpotencial $\xi$ [29],

$$
\nabla \times \mathbf{p}_{s}=0 \Rightarrow \mathbf{p}_{s}=\nabla \xi
$$

A liberdade de calibre que tem $\mathbf{A}$,

$$
\mathbf{A}^{\prime}=\mathbf{A}+\nabla \kappa,
$$

pela condição de Coulomb implica da Eq. 38 que o superpotencial deve satisfazer a relação,

$$
\xi^{\prime}=\xi+\nabla\left(\frac{e}{c} n_{s} \kappa\right)
$$

A função $\kappa$ satisfaz a equação de Laplace, e $\nabla \cdot \mathbf{p}_{s}=0$, em razão da Eq. (40). O conjunto de condições envolvendo o calibre de Coulomb e o superpotencial define a Condição de London.

\section{B. Uma Análise pela Mecânica Quântica}

Passamos agora a discutir as equações de London por argumentos de mecânica quântica aplicados ao momento canônico. Se arguimos que $\mathbf{p}_{s}$ seja nulo na ausência de um campo magnético aplicado, então o estado fundamental tem momento canônico nulo (pelo Teorema de Bloch, veja a referência [29, 41]) logo,

$$
\left\langle\mathbf{v}_{s}\right\rangle=-\frac{e}{m c} \mathbf{A} .
$$

Se a função de onda dos superelétrons é suposta rígida, ou seja, não é modificada pelo campo magnético [25, 29], e mantém a propriedade do estado fundamental que $\left\langle\mathbf{p}_{s}\right\rangle=0$ então,

$$
\mathbf{J}_{s}=n_{s} e\left\langle\mathbf{v}_{s}\right\rangle=-\frac{1}{c \Lambda} \mathbf{A}_{s} .
$$

Decorre então da relação acima imediatamente as duas equações de London. Observamos que $\dot{\mathbf{p}}_{s}=0$, é uma condição menos exigente que $\mathbf{p}_{s}=0$. A segunda implica na primeira, mas não o contrário. Embora essa última tenha uma justificativa baseada no Teorema de Bloch [25, 41], a primeira condição conduz a uma expressão para o campo elétrico $\mathbf{E}$, que é independente do calibre, enquanto que a segunda evidencia a dependência com o calibre, pois é função do potencial vetor, $\mathbf{A}$.

\section{ALGUMAS CONSIDERAÇÕES}

O primórdio da pesquisa em supercondutividade envolveu dois grandes ramos: o termodinâmico e o eletrodinâmico. A teoria de dois fluidos de Gorter e Casimir [25] estabeleceu um modelo para a descrição do comportamento termodinâmico do supercondutor, e foi superada pela teoria de transição de fase de segunda ordem aplicada à supercondutividade, por Ginzburg e Landau, no entorno da temperatura de transição, a temperatura crítica, $\mathrm{T}_{c}$.

A eletrodinâmica de Maxwell, como comentamos, não dá conta da expulsão do fluxo magnético do interior de um metal submetido a um campo magnético, como experimentalmente observada na fase supercondutora. $\mathrm{O}$ efeito Meissner-Ochsenfeld foi o ponto de partida do trabalho dos London para estender, de forma $a d h o c$, a eletrodinâmica de forma a descrever as propriedades eletromagnéticas de um metal em sua fase supercondutora.

Não sendo um teoria de primeiros princípios, a Teoria de London traz uma descrição satisfatória em alguns aspectos, mas não explica a origem dos superelétrons, tão somente justifica estes a partir da teoria dos dois fluidos, de Gorter e Casimir. Uma situação em que a descrição de London falha, envolve o problema de interface com coexistência das fases supercondutora e normal em um metal [20]. Ademais, como citado na introdução, a previsão teórica para o comprimento de penetração de London, $\lambda_{L}$, precisou considerar a correção do comprimento de coerência, $\xi_{0}$, devida a Pippard, para um melhor ajuste com os resultados experimentais.

Para construir o que designamos por densi- 
dade de Lagrangeana de London, incorporamos um vínculo associado à condição $\mathbf{p}_{s}=0$ para $\mathrm{o}$ momento canônico, ou seja, consideramos como vínculo uma relação cuja derivada temporal já conduz à primeira equação de London. É com esta equação que obtemos a segunda equação de London, aquela que dá conta do diamagnetismo extremo. A Lagrangeana de London foi estabelecida numa forma explicitamente covariante, pois objetivamos a comparação com a Lagangeana de Proca.

A comparação revelou que a Lagrangeana de London, como mostrado na subseção 2 da seção III), é uma Lagrangeana de Proca com fonte nula $\left(\mathbf{J}_{S}=0\right)$. Esta mesma comparação explicitou que a constante de acoplamento do termo de autointeração em Proca, que involve a 'massa do campo massivo', na eletrodinâmica de London está associado ao inverso do comprimento de penetração de London. Lembramos que na teoria BCS são os pares de Cooper que respondem pela supercondutividade quando se considera o acoplamento (fraco) na interação entre elétrons e fónons.

A dependência com o calibre do termo de vínculo foi considerada nas discussões da seção (IV), e mostramos lá que as equações de London, para serem alcançadas, deve-se admitir o calibre de Coulomb, e por conseguinte, a existência do superpotencial como consequência do momento canônico nulo, o que leva a uma relação entre a liberdade de calibre e o superpotencial. Esse ponto é importante porque a argumentação pela Mecânica Quântica permite inverter a sequência das equações de London, colocando a segunda equação em (7) como o principal resultado e não a primeira equação.
Disto decorre que o ponto de vista do diamagnetismo extremo é o argumento principal para a supercondutividade, e não a condutividade infinita, condição esta trazida pela primeira equação. A sustentação no contexto quântico da Eq.(6) é dependente da escolha de calibre e essa dificuldade já era percebida por Fritz London [29. É o Teorema de Bloch aplicado à função de onda no estado fundamental que justifica o resultado para o diamagnetismo extremo.

Uma investigação posterior do termo de vínculo expresso na forma de um acoplamento tensorial foi realizada, com o intuito de alcançarmos diretamente a primeira equação de London, e não a uma equação para qual ainda devéssemos tomar a derivada parcial no tempo. Mas tal tentativa ainda não se revelou frutífera, pois, primeiramente, observamos a perda da covariância explícita do vínculo, e depois, porque o vínculo deixa de apresentar uma forma simples, tratável. Entretanto, esta abordagem constitui uma perspectiva para uma investigação futura.

\section{Agradecimentos}

Os autores agradecem o acolhimento e a contribuição do Grupo Fiscampus na realização deste trabalho e ao apoio da Universidade Estadual de Feira de Santana (UEFS). A autora (Edine) agradece a bolsa da Fundação de Amparo à Pesquisa da Bahia (Fapesb) no desenvolvimento do seu projeto de pesquisa em Iniciação Científica.
[1] S. Reif-Acherman, Liquefaction of gases and discovery of superconductivity: two very closely scientific achievements in low temperature physics. Rev. Bras. Ens. Fis. 33, (2) 2601 (2011).

[2] S. Reif-Acherman, Studies of the temperature dependence of electric conductivity for metals in the Nineteenth Century: a neglected chapter in the history of superconductivity. Rev. Bras. Ens. Fis. 33, (4) 4602 (2011).
[3] J. de Nobel, The Discovery of Superconductivity. Physics Today 49, (9) 40 (1996).

[4] P.H.E. Meijer, Kamerlingh Onnes and the discovery of superconductivity. Am. J. Phys. 62, (12) 1105 (1994).

[5] B. Meilink. Proceedings of the Royal Netherlands Academy of Arts and Sciences 4, 495 (1901-1902).

[6] C. Kittel, Introduction to Solid State Physics. (8th Edition). New Jersey: John Wiley and 
Sons, Inc. (2005).

[7] R. Meservey, B.B. Schwartz, Equilibrium Properties: comparision of experimental results with predictions of the BCS theory. In: S. Parks (Editor), Superconductivity. Vol. 1. New York: Marcel Dekker, Inc. (1969).

[8] M.B.S. Costa, A.C. Pavão, Supercondutividade: um século de desafios e superação. Rev. Bras. Ens. Fis. 34, (2) 2602 (2012).

[9] P. Pureur, Supercondutividade: cem anos de desafios. Revista USP 92, 142 (2011).

[10] P.S. Branício, Introdução à Supercondutividade, suas Aplicações e a Mini-Revolução provocada pela Redescoberta do $\mathrm{MgB}_{2}$ : uma Abordagem Didática. Rev. Bras. Ens. Fis. 23, (4) 381 (2001).

[11] W. Meissner, R. Ochsenfeld, Ein neuer Effekt bie Eintritt der Supraleitfähigkeit. Die Naturwissenschaft 21, 787 (1933). Translation by A. Forrest, A new effect concerning the onset of superconductivity. Eur. J. Phys. 4, 117 (1983).

[12] A.C. Rose-Innes, E.H. Rhoderick, Introduction to Superconductivity. (2nd Edition). Oxford: Pergamon Press (1994).

[13] F. London, H. London, The Electromagnetic Equations of the Supraconductor. Proc. Royal Soc. Lond. A 149, (866) 71 (1935).

[14] F. London, H. London, Supraleitung und Diamagnetismus. Physica 2, 341 (1935).

[15] A. Mourachkine, Room-Temperature Superconductors: Introduction to Fundamentals and Applications. Cambridge: Cambridge International Science Publishing (2004).

[16] H. Éssen, M.C.N. Fiolhais, Meissner effect, diamagnetism, and classical physics - a review. Am. J. Phy. 80, (2) 164 (2012).

[17] B. Nasmith, N. Gauthier, Electric fields in steady-state superconductivity according to London theory. Am. J. Phys. 76, (9) 872 (2008).

[18] V.L. Ginzburg, L.D. Landau, On the Theory of Superconductivity. JETP 20, 1064 (1950). In: D. ter Haar, Collected Papers of L.D. Landau. Oxford: Pergamon Press (1965).

[19] L.D. Landau, On the theory of phase transitions. JETP 7, 19 (1937). In: D. ter Haar, Collected Papers of L.D. Landau. Oxford: Pergamon Press (1965).

[20] M. Tinkham, Introduction to Superconductivity. (2nd Edition). New York: McGraw-Hill (1996).

[21] L.N. Cooper, Bound Electron Pairs in a Degenerate Fermi Gas. Phys. Rev. 104, (4) 1189 (1956).

[22] J. Bardeen, L.N. Cooper, R.J. Schrieffer, Mi- croscopic Theory of Superconductivity. Phys. Rev. 106, (1) 162 (1957).

[23] NOTA 1: A energia de gap, $\Delta$, da ordem de $K T_{c}$, é a energia entre o estado fundamental e as excitações quase-partículas do sistema. A teoria BCS mostrou que mesmo uma fraca interação atrativa entre elétrons impunha uma instabilidade do estado fundamental do mar de Fermi usual do gás de elétron com relação à formação de pares de elétrons ligados ocupando estados com igual momento e spin opostos, os pares de Cooper 20.

[24] J. Bardeen, L.N. Cooper, R.J. Schrieffer, Theory of Superconductivity. Phys. Rev. 108, (5) 1175 (1957).

[25] J. Bardeen, Introduction to Superconductivity. In: S. Flëgge, Encyclopedia of Physics. Low Temperature Physics, vol. XV. Berlin: Springer-Verlag (1956).

[26] A.A. Abrikosov, On the Magnetic Properties of Superconductors of Second Group. Sov. Phys. JETP 5, (6) 1174 (1957).

[27] K. Fossheim, Superconductivity: Discoveries and Discoverers - Ten Physics Nobel Laureates Tell Their Story. Berlin: Springer-Verlag (2013).

[28] J.D. Jackson, Classical Electrodynamics. (2nd Edition). New York: John Wiley and Sons Inc. (1975).

[29] F. London, Superfluids: Macroscopic Theory of Superconductivity. Vol. 1. (2nd Revised Edition). New York: Dover Publications (1961).

[30] NOTA 2: uma dedução das equações de London que figura em alguns livros-textos, considera como ponto de partida o modelo de Drude para a condutividade elétrica, o que significa utilizar a $2^{a}$ lei de Newton. A ideia com essa argumentação é auxiliar a compreensão do que toma lugar na fase supercondutora. Entretanto, essa dedução por argumento estritamente em Maxwell não é rigorosa, pois o resultado válido para campos magnéticos dependentes do tempo é estendido ao campo magnético independente do tempo do efeito Meissner-Ochsenfeld.

[31] NOTA 3: da discussão estabelecida para as equações $(9)$ e $(10)$ segue que a derivada temporal do campo D é desprezível, e pode então ser excluída de (5). A implicação decorrente deste fato é a Eq.111), da qual se verifica que o campo B não possui dinâmica temporal. Vale lembrar aqui que ao se assumir que a derivada temporal do campo $\mathbf{D}$ é nula, as equações (2), (3), (4) e (5) tornam-se invariantes pelas transformações de Galilei (veja: M. 
Le Bellac, J.M. Lévy-Leblond. Il Nuovo Cimento 14, 217 (1973)). É por esta razão que o fenômeno da supercondutividade é essencialmente não-relativístico, como citamos no segundo parágrafo da seção III. Esta situação é designada por Lévy-Leblond e Le Bellac como o limite magnético do campo de Maxwell, onde a magnitude do campo elétrico é muito maior que a do campo de indução magnética $(\mathbf{E} \gg$ $c \mathbf{B})$.

[32] A.B. Pippard, An experimental and theoretical study of the relation between magnetic field and current in a superconductor. Proc. Roy. Soc. Lond. A 216, 547 (1953).

[33] T.E. Faber, A.B. Pippard, The penetration depth and high-frequency resistance of superconducting aluminium. Proc. Roy. Soc. Lond. A 231, 336 (1955).

[34] V.V. Schmidt, The Physics of Superconductors - Introduction to Fundamentals and Applications. P. Müller, A.V. Ustinov (Editors). Heidelberg: Springer-Verlag (1997).

[35] H. Yukawa, On the Interaction of Elementary Particles. I. Proc. Phys.-Math. Soc. Japan 19,
$712(1935)$.

[36] L.M. Brown, Hideki Yukawa and the meson theory. Physics Today 1200, (12) 55 (1986).

[37] C.M.G. Lattes, H. Muirhead, G.P.S. Occhialini, C.F. Powell, Processes involving charged mesons. Nature 159, 694 (1947).

[38] D.N. Poeranu, A. Calboreanu, Alexandru Proca (1897-1955) and his equation of the massive vector boson field. Europhysics News 37, (5) 24 (2006).

[39] F. de Aquino, Proca Equations and the Photon Imaginary Mass. hal.archivesouvertes.fr/HAL01084269 (2014).

[40] M. Tajmar, Electrodynamics in Superconductors Explained by Proca Equations. Phys. Rev. Lett. A 372, (18) 3289 (2008).

[41] D. Bohm, Note on a Theorem of Bloch Concerning Possible Causes of Superconductivity. Phys. Rev. 75, (3) 502 (1949).

[42] Um portal dedicado às notícias, novidades, e à história sobre a Supercondutividade: http://www.superconductors.org. Acessado em: 20/10/2018. 